\title{
Circadian Rhythms in the CNS and Peripheral Clock Disorders: Chronopharmacological Findings on Antitumor Drugs
}

\author{
Shigehiro Ohdo ${ }^{1, *}$ \\ ${ }^{1}$ Pharmaceutics, Division of Clinical Pharmacy, Department of Medico-Pharmaceutical Sciences, \\ Faculty of Pharmaceutical Sciences, Kyushu University, 3-1-1, Maidashi, Higashi-Ku, Fukuoka 812-8582, Japan
}

Received November 8, 2006; Accepted December 14, 2006

\begin{abstract}
The effectiveness and toxicity of antitumor drugs vary depending on dosing time associated with the 24-h rhythms of biochemical, physiological, and behavioral processes under the control of the circadian clock. Such chronopharmacological phenomena are influenced by not only the pharmacokinetics but also pharmacodynamics of medications. For example, the antitumor effect and/or toxicity of irinotecan hydrochloride, interferon, and antiangiogenic agents vary depending on the dosing time associated with the 24-h rhythm of their target enzyme, receptor, protein, and pharmacokinetics. Many of them are controlled by clock genes. Chronotherapy is especially relevant when the risk and/or intensity of the symptoms of disease vary predicably over time. In a randomized multicenter trial involving patients with previously untreated metastases from colorectal cancer, the chronomodulated infusion of oxaliplatin, fluorouracil (5-FU), and folinic acid is compared with a constant-rate infusion method. Side effects such as stomatitis, peripheral sensory neuropathy are lower and the objective response is higher in the chronotherapy as compared with the fixed-rate infusion. The merit of chronomodulated infusion is supported by the 24-h rhythm of DNA synthesis and the activity of dehydropyrimidine dehydrogenase, which brings about the intracellular catabolism of 5-FU. Although interferon (IFN) also alters the clock function, the disruptive effect of IFN on clock function can be overcome by devising a dosing regimen that minimizes adverse drug effects on clock function. Thus one approach to increasing the efficiency of pharmacotherapy is the administration of drugs at times at which they are most effective and/or best tolerated.
\end{abstract}

Keywords: chronopharmacology, chronotherapy, chronopharmacokinetics, circadian rhythm, clock gene

\section{Introduction}

Many drugs vary in potency and/or toxicity associated with the rhythmicity of biochemical, physiological, and behavioral processes $(1-5)$. Theoretically, it has been argued that drug administration at certain times of the day should improve the outcome of pharmacotherapy. The knowledge of 24-h rhythm in the risk of disease plus evidence of 24-h rhythm dependencies of drug pharmacokinetics, effects, and safety constitutes the rationale for pharmacotherapy. One approach to increasing the efficiency of pharmacotherapy is the administra-

*Corresponding author. ohdo@phar.kyushu-u.ac.jp

Published online in J-STAGE: February 14, 2007

doi: 10.1254/jphs.FMJ06003X6 tion of drugs at times at which they are most effective and/or best tolerated. The application of biological rhythm to pharmacotherapy may be accomplished by the appropriate timing of conventionally formulated tablets and capsules and the use of a special drug delivery system to synchronize drug concentrations to rhythms in disease activity.

Several drugs are not only influenced by biological rhythms, but also act on the biological rhythms (6). On the horizon are drugs to fix broken biological clocks, perhaps a factor in all illnesses, in the opinion of some physicians. In contrast, several drugs cause alterations in the 24-h rhythms of biochemical, physiological, and behavioral processes. The alteration of rhythmicity is sometimes associated with therapeutic effects (i.e., anti- 
depressant drugs) or instead may lead to illness and altered homeostatic regulation.

In all living organisms, one of the most indispensable biological functions is the circadian clock (suprachiasmatic nuclei: $\mathrm{SCN}$ ), which acts like a multifunctional timer to regulate homeostatic systems such as sleep and activity, hormone levels, appetite, and other bodily functions with 24-h cycles (7). Recently, clock genes are identified as the genes that ultimately control a vast array of circadian rhythms in physiology and behavior $(8,9)$. The knowledge of clock genes may be important for clinical practice. The aim of this review is to provide an overview of the dosing time-dependent alterations in effects and safety of antitumor drugs.

\section{Chronopharmacological study in animal}

Dosing time influences the extent of toxicity of many antitumor drugs, including cytostatics and cytokines, in mice and rats (10). Dosing time not only affects drug tolerability, but may also modify antitumor efficacy. Chronopharmacologic mechanisms may involve 24-h rhythm in drug pharmacokinetics and/or susceptibility rhythms of target tissues. Plasma and urinary chronopharmacokinetics have been investigated for several antitumor drugs. The 24-h rhythm in cellular enzymatic activities also account in part for the chronopharmacology of antimetabolites. This is the case for dihydrofolate reductase, a target enzyme for methotrexate cytotoxicity; dehydropyrimidine dehydrogenase, the rate-limiting catabolic enzyme of fluoropyrimidines; uridine phosphorylase; orotate phosphoribosyltransferase; and deoxythymidine kinase - all three involved in the anabolism of the cytotoxic forms of fluoropyrimidines. The examples of chronopharmacological findings are described below.

Biological rhythms at the cellular and subcellular level can give rise to significant dosing-time differences in the pharmacodynamics of medications that are unrelated to their pharmacokinetics. This phenomenon is termed chronesthesy. Rhythms in the receptor number or conformation, second messengers, metabolic pathways, and/or free-to-bound fraction of medications help to explain this phenomenon. For example, the antitumor effect of interferon (IFN)- $\beta$ and the antiviral effect and lymphocyte stimulating effect of IFN- $\alpha$ in mice are more efficient during the early rest phase than during the early active phase $(11,12)$. The dosing schedule-dependent effect of IFN $-\beta$ or IFN- $\alpha$ is also closely related to those of IFN receptors and ISGF expression in tumor cells or lymphocytes.

The term chronotoxicity refers specifically to predictable-in-time variation in patient vulnerability to the side effects of medications due to biological rhythm determinants. Chronotoxicities are known especially for antitumor agents. For example, the body weight loss induced by irinotecan hydrochloride (CPT-11) in nocturnally active mice is more serious in the late active phase and the early rest phase and milder in the late rest phase and the early active phase (13). The CPT-11induced leukopenia is more serious in the late active phase and milder in the late rest phase. The lower toxicity of CPT-11 is observed when DNA synthesis and type I DNA topoisomerase activity in bone marrow cells decrease and the higher toxicity is observed when these activities begin to increase. The finding indicates that the choice of dosing time associated with the 24-h rhythm of DNA synthesis may help to achieve a rational chronotherapeutic strategy, reducing the toxic effects of CPT-11 and/or increasing its therapeutic effects. Cell division in many mammalian tissues is associated with specific times of day. In the regenerating liver of mice, the circadian clock controls the expression of cell cyclerelated genes that in turn modulate the expression of active Cyclin B1-Cdc2 kinase, a key regulator of mitosis (14). Among these genes, expression of weel is directly regulated by the molecular components of the circadian clockwork. On the other hand, the circadian clockwork oscillates independently of the cell cycle in single cells. The intracellular circadian clockwork can control the cell-division cycle directly and unidirectionally in proliferating cells. Thus, the regulatory mechanisms underlying 24-h rhythm of pharmacodynamics should be also clarified from the viewpoints of clock genes.

Angiogenesis is important for tumor growth and metastasis. Hypoxia-induced expression of vascular endothelial growth factor (VEGF) plays an important role in tumor-induced angiogenesis. The levels of $V E G F$ mRNA in tumor cells implanted in mice rise substantially in response to hypoxia, but the levels show a 24-h rhythm (15). Luciferase reporter gene analysis reveals that Per2 and Cry1, whose expression in the implanted tumor cells shows a 24-h rhythm, inhibit the hypoxiainduced $V E G F$ promoter activity. Namely, the negative limbs of the molecular loop periodically inhibit the hypoxic induction of $V E G F$ transcription, resulting in the 24-h fluctuation of its mRNA expression. Furthermore, the antitumor efficacy of antiangiogenic agents is enhanced by administering the drugs at the time when VEGF production increases.

Methionine aminopeptidase2 (MetAP2) plays an important role in the growth of endothelial cells during the tumor angiogenesis stage. MetAPs show a 24-h rhythm in implanted tumor masses (16). The mechanism underlying the 24-h rhythm of MetAP2 activity is investigated in tumor-bearing mice. The $5^{\prime}$ flanking 
region of MetAP2 includes eight E-boxes. The transcription of the MetAP2 promoter is enhanced by the CLOCK:BMAL1 heterodimer, and its activation is inhibited by PER2 or CRY1. Deletion and mutation of the E-boxes in the region indicate that the E-box nearest to the initiation start site plays an important role in the transcriptional regulation by clock genes. In sarcoma180-bearing mice, the pattern of binding of CLOCK and BMAL1 to the E-box and transcription of the MetAP2 promoter shows a 24 -h rhythm with higher levels from the mid-light to early-dark phase. MetAP2 protein expression varies with higher levels from the late-dark to early-light phase. Namely, the 24-h rhythm of MetAP2 activity is regulated by the transcription of clock genes within the clock feedback loops. Furthermore, the antitumor efficacy of MetAP2 inhibitor is enhanced by administering the drugs at the time when MetAP2 activity increases.

\section{Relevance of chronopharmacological concepts in clinical practice}

The chronobiology and chronopharmacological concepts to drug efficacy and safety have been exemplified in terms of drug testing and post-approval drug surveillance.

Chronotoxicities are known especially for antitumor agents $(10,17,18)$. In a randomized multicenter trial involving patients with previously untreated metastases from colorectal cancer, 93 patients were assigned chronotherapy and 93 were assigned constant-rate infusion via multichannel programmable ambulatory pumps $(10,17)$. The chronomodulated infusion of oxaliplatin (peak at 16:00), 5-fluorouracil (5-FU) (peak at 04:00), and folinic acid (peak at 04:00) was compared with a constant-rate infusion method. Severe stomatitis was seen in $76 \%$ of the patients receiving fixed-rate infusion and in $14 \%$ of those on chronotherapy. Cumulative peripheral sensory neuropathy with functional impairment is reported in $31 \%$ patients on constant delivery and in $16 \%$ patients on chronotherapy. Objective response rate is $51 \%$ for chronotherapy and $29 \%$ for constant-rate delivery. According to this multicenter randomized trial, therefore, the most active chronomodulated schedule is also the least toxic one. Median survival is 16 months in both modalities, possibly because $24 \%$ of the patients crossed over from the flat schedule to chronotherapy. The merit of chronomodulated infusion described above is supported by the following evidences. In human bone marrow, skin, and oral and rectal mucosae, DNA synthesis, a stage of the cell-division cycle associated with increased susceptibility to S-phase-specific agents, decreases by $50 \%$ or more between 00:00 and 04:00 compared with daytime (10). The activity of dehydropyrimidine dehydrogenase in human mononuclear cells increases by $40 \%$ around midnight (10). This enzyme brings about the intracellular catabolism of 5-FU and contributes to improved tolerability of this drug between 00:00 and 04:00.

Two clinical trials compared the toxicity of two dosing times of anthracyclines and cisplatin in 30 patients with advanced ovarian cancer. Both studies demonstrated that doxorubicin is better tolerated near 06:00 and cisplatin between 16:00 and 20:00 than 12-hapart $(10,18)$. The chronopharmacokinetic properties of cisplatin seem to contribute to the decreased renal toxicity during evening administration. These findings show that the circadian stage at which antitumor drugs are given to patients should be carefully considered. One approach to increasing the efficiency of pharmacotherapy is administering drugs at times during which they are best tolerated.

\section{Dosing time-dependent alteration of clock function}

IFNs have been widely used as antiviral and antitumor agents. However, IFNs cause adverse neuropsychiatric effects such as depression and neurosis and they are reported to sometimes lead to suicide $(19,20)$. When IFNs are administered during the early active phase in diurnally active humans, alterations in the 24-h rhythm are suggested by the changes in the lymphocyte counts and cortisol levels (21). However, the mechanism has not been clarified from the viewpoint of the disruptive effect of the drug on the clock genes. Recently we clarify the disruptive effect of IFN- $\alpha$ on the rhythm of $m P e r$ genes mRNA expression in the SCN of mice $(22,23)$. These findings are supported by the inhibitory effect of IFN- $\alpha$ on the mRNA expression of mClock and $m B m a l l$, which are important factors in activating the transcription of mPers, vasopressin, and the $m D b p$ gene showing specific output function from the SCN to the periphery (9). Interestingly, an inhibitory effect of mRNA expression of each clock gene in the SCN is observed by the repetitive administration of IFN- $\alpha$ during the early active phase, but not the early rest phase. The observations for humans described above correspond well to the findings indicating that alteration of the clock genes is induced by IFN- $\alpha$ administration during the early active phase in nocturnally active rodents. Thus, alteration of the clock function may be overcome by devising a dosing regimen that minimizes adverse drug effects on clock function. 


\section{Conclusions}

The effectiveness and toxicity of antitumor drugs vary depending on dosing time associated with 24-h rhythms of biochemical, physiological, and behavioral processes under the control of the circadian clock. The knowledge of the 24-h rhythm in the risk of disease plus evidence of 24-h-rhythm dependencies of drug pharmacokinetics, effects, and safety constitutes the rationale for pharmacotherapy. To monitor the rhythmicity of pharmacokinetics and/or pharmacodynamics, it may be useful to choose the most appropriate time of day for administration of drugs that may increase their therapeutic effects and/or reduce their side effects. In contrast, several drugs cause alterations in the 24-h rhythms of biochemical, physiological, and behavioral processes. Attention should be paid to the alteration of clock genes expression, and this should be considered as an adverse effect when it leads to altered regulation of the circadian system that is a serious problem affecting basic functioning of living organisms. The alteration of the clock function, a new concept of adverse effects, can be overcome by devising a dosing regimen that minimizes adverse drug effects on clock function.

\section{References}

1 Halberg F. Chronobiology. Annu Rev Physiol. 1969;31:675725.

2 Reinberg A, Halberg F. Circadian chronopharmacology. Annu Rev Pharmacol. 1971;11:455-492.

3 Labrecque G, Belanger PM. Biological rhythms in the absorption, distribution, metabolism and excretion of drugs. Pharmacol Ther. 1991;52:95-107.

4 Haus E, Touittou Y. Chronobiology of development and aging. In: Redfern PH, Lemmer B, editors. Physiology and pharmacology of biological rhythms. Berlin Heidelberg: Springer-Verlag; 1997. p. 95-134.

5 Smolensky MH, Labrecque G. Chronotherapeutics. Pharmaceutical News. 1997;4:10-16.

6 Duncan WC. Circadian rhythms and the pharmacology of affective illness. Pharmacol Ther. 1996;71:253-312.

7 Moore RY, Eichler VB. Loss of a circadian adrenal corticosterone rhythm following suprachiasmatic lesions in the rat. Brain Res. 1972;42:201-206.
8 Tei H, Okamura H, Shigeyoshi Y, Fukuhara C, Ozawa R, Hirose $\mathrm{M}$, et al. Circadian oscillation of a mammalian homologue of the Drosophila period gene. Nature. 1997;389:512-516.

9 Chang DC, Reppert SM. The circadian clocks of mice and men. Neuron. 2001;29:555-558.

10 Levy F. Circadian chronotherapy for human cancers. Lancet Oncology. 2001;2:307-315.

11 Takane H, Ohdo S, Yamada T, Yukawa E, Higuchi S. Chronopharmacology of antitumor effect induced by interferon- $\beta$ in tumor-bearing mice. J Pharmacol Exp Ther. 2000;294:746-752.

12 Ohdo S, Wang DS, Koyanagi S, Takane H, Inoue K, Aramaki H, et al. Basis for dosing time-dependent changes in the antiviral activity of interferon- $\alpha$ in mice. J Pharmacol Exp Ther. 2000;294:488-493

13 Ohdo S, Makinosumi T, Ishizaki T, Yukawa E, Higuchi S, Nakano $S$, et al. Cell cycle-dependent chronotoxicity of irinotecan hydrochloride in mice. J Pharmacol Exp Ther. 1997;283:1383-1388.

14 Matsuo T, Yamaguchi S, Mitsui S, Emi A, Shimoda F, Okamura $\mathrm{H}$. Control mechanism of the circadian clock for timing of cell division in vivo. Science. 2003;302:255-259.

15 Koyanagi S, Kuramoto Y, Nakagawa H, Aramaki H, Ohdo S, Soeda $\mathrm{S}$, et al. A molecular mechanism regulating circadian expression of vascular endothelial growth factor in tumor cells. Cancer Res. 2003;63:7277-7283.

16 Nakagawa H, Koyanagi S, Takiguchi T, Kuramoto Y, Soeda S, Shimeno $\mathrm{H}$, et al. 24-hour oscillation of mouse methionine aminopeptidase2, a regulator of tumor progression, is regulated by clock gene proteins. Cancer Res. 2004;64:8328-8333.

17 Levi F, Zidani R, Misset JL. Randomised multicentre trial of chronotherapy with oxaliplatin, fluorouracil, and folinic acid in metastatic colorectal cancer. Lancet. 1997;350:681-686.

18 Hrushesky WJM. Circadian timing of cancer chemotherapy. Science. 1985;228:73-75.

19 Fattovich G, Giustina G, Favarato S, Ruol A. A survey of adverse events in 11241 patients with chronic viral hepatitis treated with alfa interferon. J Hepatol. 1996;24:38-47.

20 Jansenn HLA, Brouwer JT, Mast RC, Schalm SW. Suicide associated with alfa-interferon therapy for chronic viral hepatitis. J Hepatol. 1994;21:241-243.

21 Bocci V. Administration of interferon at night may increase its therapeutic index. Cancer Drug Delivery. 1985;2:313-318.

22 Ohdo S, Koyanagi S, Suyama H, Higuchi S, Aramaki H. Changing the dosing schedule minimizes the disruptive effects of interferon on clock function. Nature Med. 2001;7:356-360.

23 Koyanagi S, Ohdo S. Alteration of intrinsic biological rhythms during interferon treatment and its possible mechanism. Mol Pharmacol. 2002;62:1393-1399. 